\title{
Simulating the human body's microclimate using automatic coupling of CFD and an advanced thermoregulation model
}

\author{
Conrad Voelker, Hayder Alsaad \\ Bauhaus-University Weimar, Department of Building Physics \\ Coudraystrasse 11A, Room 110 \\ 99423 Weimar, Germany \\ Email:conrad.voelker@uni-weimar.de,hayder.alsaad@uni-weimar.de \\ (Received 27 June 2017; accepted 22 January 2018; first published 02 February 2018)
}

\section{Copyright Notice}

This is the peer reviewed version of the article published in Indoor Air 28 (3), 415-425 (2018) and may be found in final form at https://doi.org/10.1111/ina.12451. This article may be used for non-commercial purposes in accordance with Wiley Terms and Conditions for Self-Archiving.

Copyright 2018 John Wiley \& Sons. This article may be downloaded for personal use only. Any other use requires prior permission of the authors and John Wiley \& Sons.

\begin{abstract}
This study aims to develop an approach to couple a computational fluid dynamics (CFD) solver to the University of California, Berkeley (UCB) thermal comfort model to accurately evaluate thermal comfort. The coupling was made using an iterative Java script to automatically transfer data for each individual segment of the human body back and forth between the CFD solver and the UCB model until reaching convergence defined by a stopping criterion. The location from which data are transferred to the UCB model was determined using a new approach based on the temperature difference between subsequent points on the temperature profile curve in the vicinity of the body surface. This approach was used because the microclimate surrounding the human body differs in thickness depending on the body segment and the surrounding environment. To accurately simulate the thermal environment, the numerical model was validated beforehand using experimental data collected in a climate chamber equipped with a thermal manikin. Furthermore, an example of the practical implementations of this coupling is reported in this paper through radiant floor cooling simulation cases, in which overall and local thermal sensation and comfort were investigated using the coupled UCB model.
\end{abstract}




\section{Introduction}

Occupants' health and performance is greatly affected by their thermal comfort state in the indoor environment. The indoor environment is affected by heat released from the occupants and the interaction between the microclimate around the body and the surrounding ventilation flow. ${ }^{1-4}$ Therefore, it is necessary to understand the characteristics of the air in the vicinity of the human body. Due to convective heat release, the human body is encased in a microclimate that consists of a boundary layer with significantly different air temperature and velocity from that of the surrounding environment. Under typical conditions, the convective heat dissipation from the human body is dominated by buoyancydriven natural convection. In this case, the temperature difference between the surface of the human body and the adjacent air is the most influential variable on the properties of the microclimate. This difference in temperature determines the velocity of the flow responsible for the convective heat transfer. The higher the temperature difference, the higher the air velocity in the microclimate and the higher the heat transfer coefficient of the body segments. ${ }^{5}$ Furthermore, other factors such as clothing, body geometry (posture, shape and size), ventilation and human respiratory flow have a major influence on the thickness of the microclimate around the human body as well.6,7 Furniture type and arrangement such as the chair design and table positioning in front of the body also are found to have an impact on the characteristics of the microclimate.8,9 Thus, the thickness of the human convective boundary layer is different depending on the indoor climate.

The shape and size of the body segments also influence the attributes of the microclimate. Generally, the thickness of human convective boundary layer at the feet region is relatively small (around $5 \mathrm{~cm}$ ) as stated by Murakami et al.10 The thickness of this layer increases as one moves upwards along the body surface. The thickness grows around large surfaces along the flow direction, such as the chest and the back, where convective heat loss is low. Nineteen-cm thickness was measured at the neck. ${ }^{10}$ Moreover, the thickness of the layer defined by the flow velocity might be different from the thickness of the thermal layer. The thickness of the flow velocity-defined layer ranges from $8 \mathrm{~cm}$ around the feet to 15 $\mathrm{cm}$ at the head. The maximum flow velocity is about 0.2-0.3 $\mathrm{m} / \mathrm{s}$, which can be found within the plume above the head.11

The coupling of computational fluid dynamics (CFD) and a human thermoregulation model accounts for the different characteristics of the human body's microclimate when evaluating the thermal environment. It offers potential for determining both thermal comfort and the interaction between occupants and their surrounding environment. One of the first attempts to couple CFD and a human thermoregulation model was developed by Maué et al. ${ }^{12}$ in which Stolwijks' thermoregulation model was linked to the commercial software Star-CCM+ to investigate thermal comfort in automobiles. Another study at the University of
Tokyo coupled Gagge's 2-node model with CFD simulations. This study implemented a highly simplified geometry of the occupant due to an inadequate amount of computational power at the time.13 Tanabe et al. used a substantially improved geometry in the coupling with a self-developed thermoregulation model.14 The "Tanabe model" was also implemented by Bartsch and Spille-Kohoff ${ }^{15}$ with the commercially available CFD software ANSYS CFX. Streblow et al. further developed the Tanabe model based on their own empirical measurements, their developed model was then coupled with ANSYS CFX. ${ }^{16}$ Another thermoregulation model ("Fiala model") was linked to ANSYS CFX at Loughborough University. ${ }^{17}$ A promising research was conducted by van Treeck $^{18}$ in which the coupling with the Fiala model was performed using high performance computers that can produce results in almost real-time. This allows interactive control of the indoor environment in future applications. Huang investigated thermal comfort in automobiles by coupling CFD simulations with the University of California, Berkeley (UCB) model. ${ }^{19}$ Further coupling with the UCB model was conducted at the Hong Kong Polytechnic University which focused on personalized ventilation (PV), ${ }^{20,21}$ in which data transfer was carried out manually.

This study also implements the UCB model, which was developed based on the Stolwijk model to evaluate complex thermal environments. This UCB thermal comfort model is a system of linked non-linear differential equations that describe the human body's heat balance. It consists of 16 body segments, each consisting of four concentric layers (core, muscle, fat, and skin). ${ }^{22}$ Furthermore, the model implements an additional node that accounts for heat and moisture transfer through the clothing. ${ }^{23}$ Heat transfer by convection, conduction, and radiation (short-wave as well as long-wave) is calculated independently. Additionally, the model utilizes a detailed approach to calculate heat exchange due to blood flow. It accounts for physiological mechanisms and factors such as vasodilation, vasoconstriction, sweating, metabolic rate, gender, and age.22 Moreover, the model recognizes individual physiognomic differences and their influence on the thermoregulation of the body. ${ }^{24}$ As a result of its comprehensive components, the UCB model has received much attention; ${ }^{25}$ It was used by many researchers to evaluate thermal comfort due to its comprehensive method of calculation. ${ }^{26-28}$

Even though coupling with CFD simulations has been investigated by researchers before, there is still a shortage of information about this topic in literature. The details of the preparatory work, validation, and clear data about the boundary conditions must be studied and presented. Furthermore, since the human body's microclimate does not have a uniform thickness, the location from which air temperature in the macroclimate is transferred to the thermoregulation model must be determined. This paper aims to contribute to existing knowledge by addressing these issues.

This study introduces an automatic coupling of the UCB thermal comfort model and Star-CCM+CFD software using a 

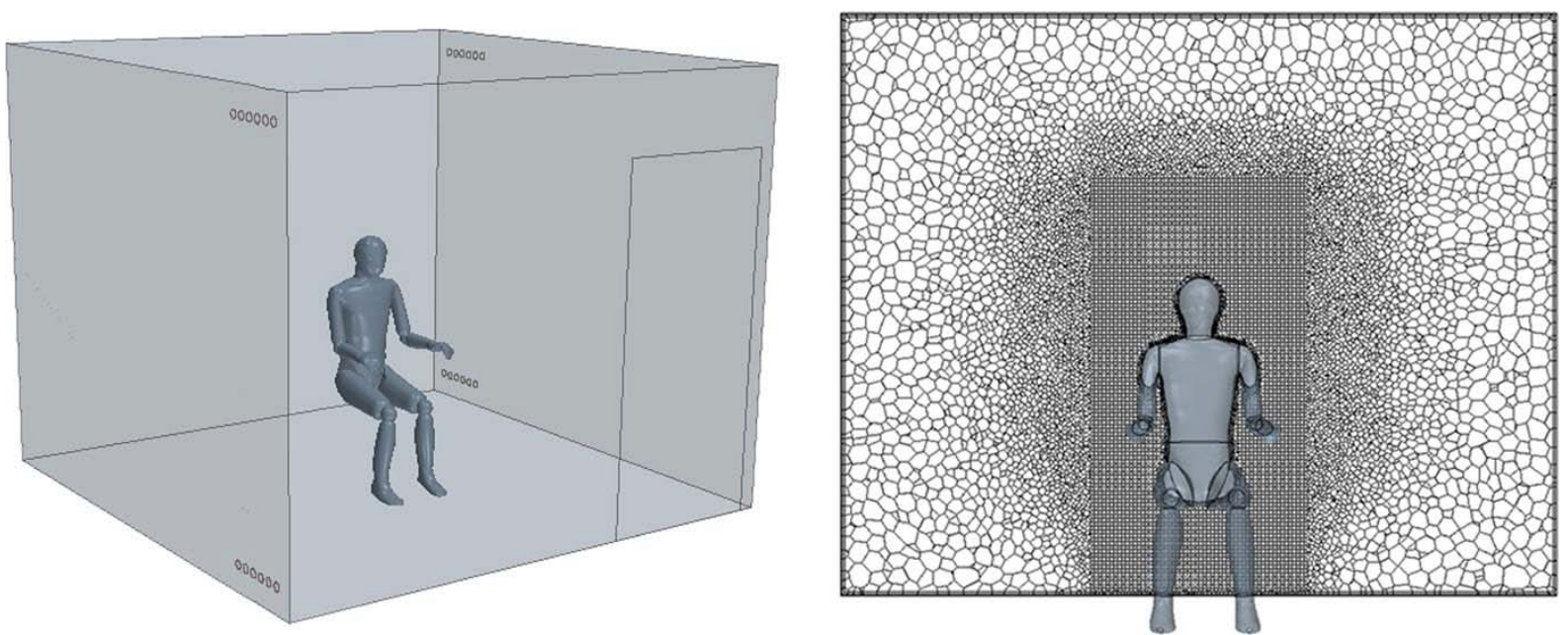

Figure 1 Left: the geometry of the numerical model; right: Front view of the polyhedral mesh with fine cells around the manikin model

simple yet practical scheme. Before developing the coupling, a series of empirical measurements was conducted in a climate chamber. The CFD model was then validated using data from the empirical measurements. Afterwards, a Java script was programmed to automatically couple the CFD simulation to the UCB thermal comfort model. This script transfers data back and forth between the CFD solver and the UCB model until it reaches convergence defined by a stopping criterion.

\section{Measurements and validation}

To validate the numerical model, a series of measurements was performed in the climate chamber of the Department of Building Physics at the Bauhaus-University Weimar. The chamber is $3 \times 3 \times 2.44 \mathrm{~m}$ situated inside a laboratory hall to isolate it from the outdoor environment. In addition to its ventilation system, the chamber can be tempered by controlling the temperature of each interior surface ( 4 walls, floor, and ceiling) separately through waterbearing capillary tubes embedded under the finishing layer. In this study, the ventilation system was turned off; the climate chamber was tempered by setting all the six surfaces to the same temperature $\left(\theta_{w}=18^{\circ} \mathrm{C}\right)$. This resulted in a relatively uniform thermal environment with almost no vertical stratification and no radiant temperature asymmetries. Moreover, this approach simplifies the assessment of thermal comfort since mean radiant temperature is approximately equal to air temperature in this case.

The chamber is equipped with a thermal manikin with a complex male body shape that simulates the heat release of the human body. The manikin's body is $1.23 \mathrm{~m}$ high in the upright sitting posture; it consists of 22 body segments. The manikin was situated naked at the centre of the chamber, and seated on a backless, seatless chair frame to allow for air movement. The breathing function of the manikin was turned off to simplify both measurements and CFD simulation. The temperature of each segment of the manikin's body was determined using the UCB thermal comfort model under default settings ( 0 clo and 1 met).

Twenty-five Ahlborn's (Holzkirchen, Germany) negative temperature coefficient (NTC) temperature sensors with an accuracy of $\pm 0.1 \mathrm{~K}$ were placed around the manikin to measure the air temperature in the microclimate. Air velocity was measured using Ahlborn's omni-directional hotwire anemometers with an accuracy of $\pm 1.5 \%$ of the measured values. Measurements were conducted for a period of $t=1 \mathrm{~h}$, the sampling interval was $t=1 \mathrm{~s}$. Data were recorded and averaged, and statistical fluctuations were calculated. This allowed comparison to the simulation results, because CFD steady state simulation implements Reynolds-averaged Navier-Stokes (RANS) equations that statistically average fluctuations in the flow. A full description of the measurements and results can be found in the published work of Voelker et al. 5

To make the simulation results comparable to the measurements, it is necessary to conduct the simulation using a detailed geometry of the chamber and the measurements setup. Therefore, a 3d laser scanner was used to capture the exact shape of the thermal manikin (Figure 1). Star-CCM+ software was used to run the CFD simulation in this research. To simulate natural convection, the air domain was set as an incompressible ideal gas. This assumption justifies the use of the incompressible Navier-Stokes equations. Moreover, Boussinesq approximation was used to treat the effect of temperature on density. The numerical model consisted of $\sim 1,600,000$ polyhedral cells with a cell base size of $7 \mathrm{~cm}$ with a local mesh refinement around the manikin's body of $75 \%$ of the cell size to achieve a y+ value of $\sim 1$ (Figure 1, right). Similar to the empirical measurements, the temperature of the six surfaces of the climate chamber geometry were set to $\theta_{w}=$ $18^{\circ} \mathrm{C}$. The surface temperature of the manikin's body segments used in the empirical measurements was defined in the CFD solver. Since all surfaces in the numerical model were defined as fixed temperature boundary condition, radiative heat transfer was not accounted for in the CFD simulation. Relative 


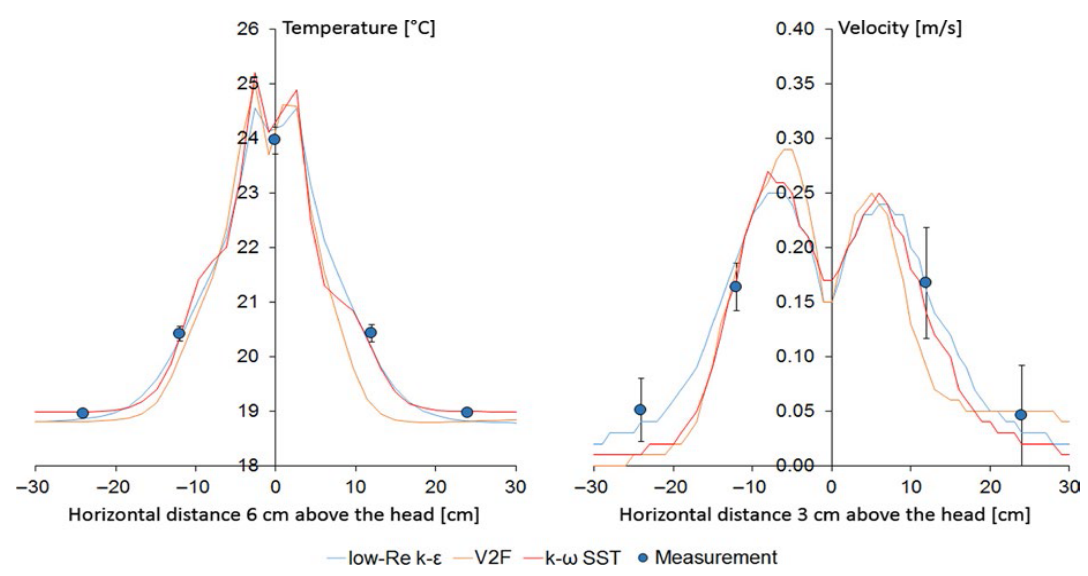

Figure 2 Simulated and measured air temperature and velocity horizontally above the head, where 0 is manikin's symmetry plane

humidity was not considered in the CFD simulation, and it was left to its default value $(\varphi=50 \%)$ in the UCB model.

Validation was conducted by comparing air temperature and velocity between measurements and simulation in multiple points around the manikin's body. An example of this is presented in Figure 2, which exhibits measured and simulated air temperature and velocity horizontally above the head. Error bars plotted in Figure 2 indicate the standard deviation of the measured values. After comparing all of the available turbulence models, the k- $\omega$-SST model showed the best agreement with the measured data. The k- $\omega$-SST model utilizes the k- $\varepsilon$ model within the free space and the k- $\omega$ model near the walls, which combines the advantages of the two models in a blending function. Therefore, the coupling was conducted using this turbulence model.

\section{Coupling of CFD and UCB model}

As mentioned earlier, the CFD solver and the UCB model were linked using a Java script. The CFD solver calculates the climatic conditions around the manikin, and subsequently transfers these conditions to the UCB model. The process starts with initial conditions, which need to be chosen by the user. Afterwards, air temperature $\theta_{a}$ and heat transfer coefficients $h_{c}$ are automatically taken to the UCB model from the CFD solver after each iteration for each body segment. Based on this, the UCB model determines the segment-specific surface temperature of the human body; data are then transferred back to the CFD solver in an iterative process since heat emission from the body influences the room climate (Figure 3).

To end this iteration loop, a stopping criterion was defined as the amount of difference between the results of two subsequent loops of the iteration. $\varepsilon \leq 0.1 \mathrm{~K}$ was selected as a stopping criterion for the coupling of the two already converged simulations. In other words, before coming to this criterion, both CFD and UCB simulations were fully converged. Multiple test simulations were conducted when the coupling script was developed to ensure the sensitivity of the selected criterion. Furthermore, a $0.1 \mathrm{~K}$ temperature difference has no significant influence on the calculated thermal sensation and comfort in the UCB model. Numerous tests of the coupling showed that 3-4 iteration loops were required to reach this criterion and achieve convergence. When the simulation is finished, local thermal sensation was determined by the UCB model as a function of local and overall averaged skin temperature using a logistical function. The determination of the thermal comfort took place in the UCB model depending on the local and overall thermal sensation.25,29-31

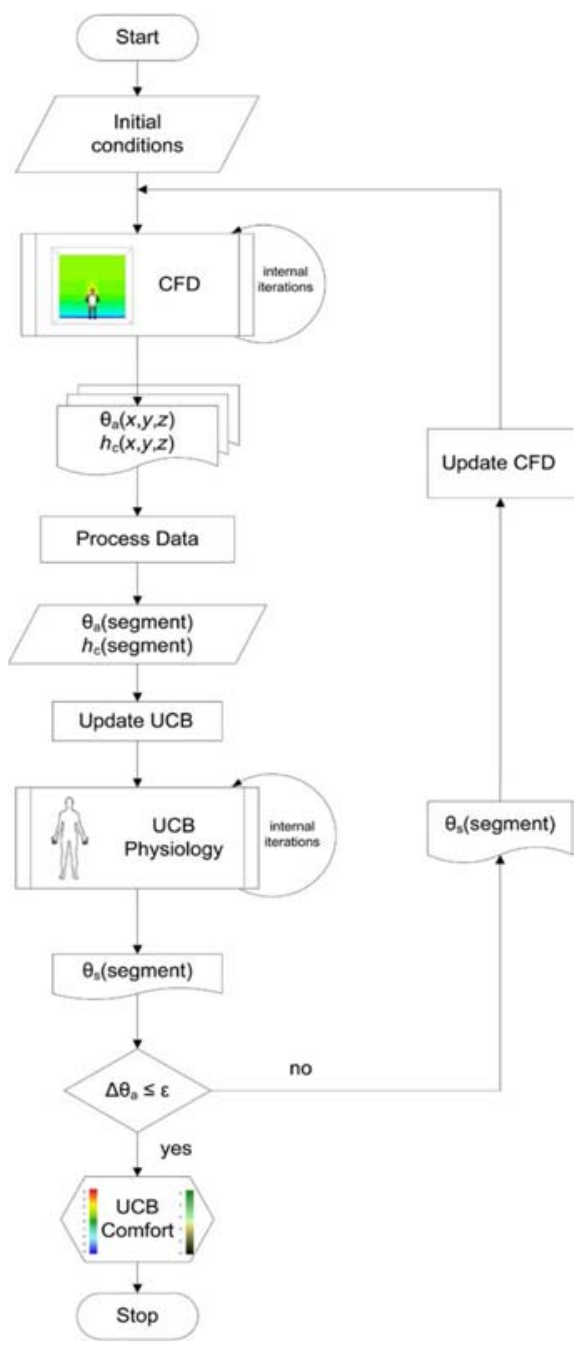

Figure 3 CFD and UCB coupling flowchart 


\subsection{The microclimate around the human body}

We define the microclimate as the range in which the properties of the flow differ from the air in the macroclimate by the presence of a human being. Thus, the definition is similar to Ludwig Prandtl's concept of "boundary layer," in which va,microclimate $\leq 0.99 \cdot v a$,macroclimate. ${ }^{32}$ To transfer the climatic parameters from the CFD simulation to the UCB model, an exact knowledge of the microclimate surrounding the human body is necessary.

Figure 4 (left) shows the simulated air temperature in the microclimate. It is noticeable that the air temperature in the microclimate is significantly higher than the room temperature due to the heat dissipation of the human body via convection. This temperature gradient is responsible for a density gradient, which is the prerequisite for mass transport in the case of natural convection. While the thermal microclimate is distinctly thin in the region of the lower torso, its thickness expands as the altitude increases. This expansion is significant at the change in the body geometry at the shoulder and neck. In addition, the head is an obstacle in the field of the buoyancy-driven flow resulting in a relatively thick thermal boundary layer. The highest air temperature is found in this area of the microclimate. Besides, an ascending plume is found over the head.

Figure 4 (right) shows the simulated air velocity in the microclimate, which exhibits similar, yet not identical properties to the air temperature pattern in the microclimate. In close proximity of the human body, the velocity of the flow is generally low due to shear stress. While air velocities in the macroclimate can be seen to be near $0 \mathrm{~m} / \mathrm{s}$, the air velocity in the near-body microclimate increases to about $0.1 \mathrm{~m} / \mathrm{s}$. Similar to the thermal microclimate, the flow velocity increases significantly at the tapering geometry in the shoulder/neck region. The flow velocity reaches its maximum of $0.3 \mathrm{~m} / \mathrm{s}$ at the head as well as at the plume over the head. Interestingly, the flow reaches a break-off point after the maximum thickness at the head (supercritical flow). This so-called dead zone is characterized by small-scale swirling, which is resulted by high Reynolds and Grashof numbers in this region. Transition to the macroclimate

Air temperature around each body segment needs to be transferred to the UCB comfort model from the CFD simulation. Since the UCB model requires air temperature from the macroclimate, the transferred values should be taken from outside the microclimate far from the manikin body. Nevertheless, it should also be taken as close as possible to the corresponding body part to accurately estimate the local sensation. Therefore, it is essential to determine the thickness of the microclimate first since it differs depending on the boundary conditions and the respective segment.

Several approaches have been used in literature to transfer the values of air temperature in the macroclimate to the thermoregulation model during the coupling process. A fixed distance of $10 \mathrm{~cm}$ from the surface was used by Gao et al. ${ }^{20}$ to determine the value of air temperature in the macroclimate around each body segment for each boundary condition. This approach can be inaccurate in some cases because it neglects the case-dependent distinctive characteristics of the microclimate.

Another approach was chosen by Cropper et al. ${ }^{33}$ in which the heat flux $q$ is transferred to the thermoregulation model instead of air temperature in the macroclimate. Even though this approach is advantageous because the unknown microclimate thickness $s$ is not required, it does not provide the necessary climatic parameters required by the existing thermoregulation models. Furthermore, there are disadvantages of using heat flux to define the boundary conditions instead of temperature. Primarily, assigning heat flux does not exactly specify the respective portions of the heat emission (convective, radiative, and evaporative) which are dependent on the boundary conditions. In addition, defining the boundary conditions of each body segment in the CFD solver using heat flux can lead to inaccuracies. Figure 5 shows the simulation results when a typical convective heat flux of $q_{c}$
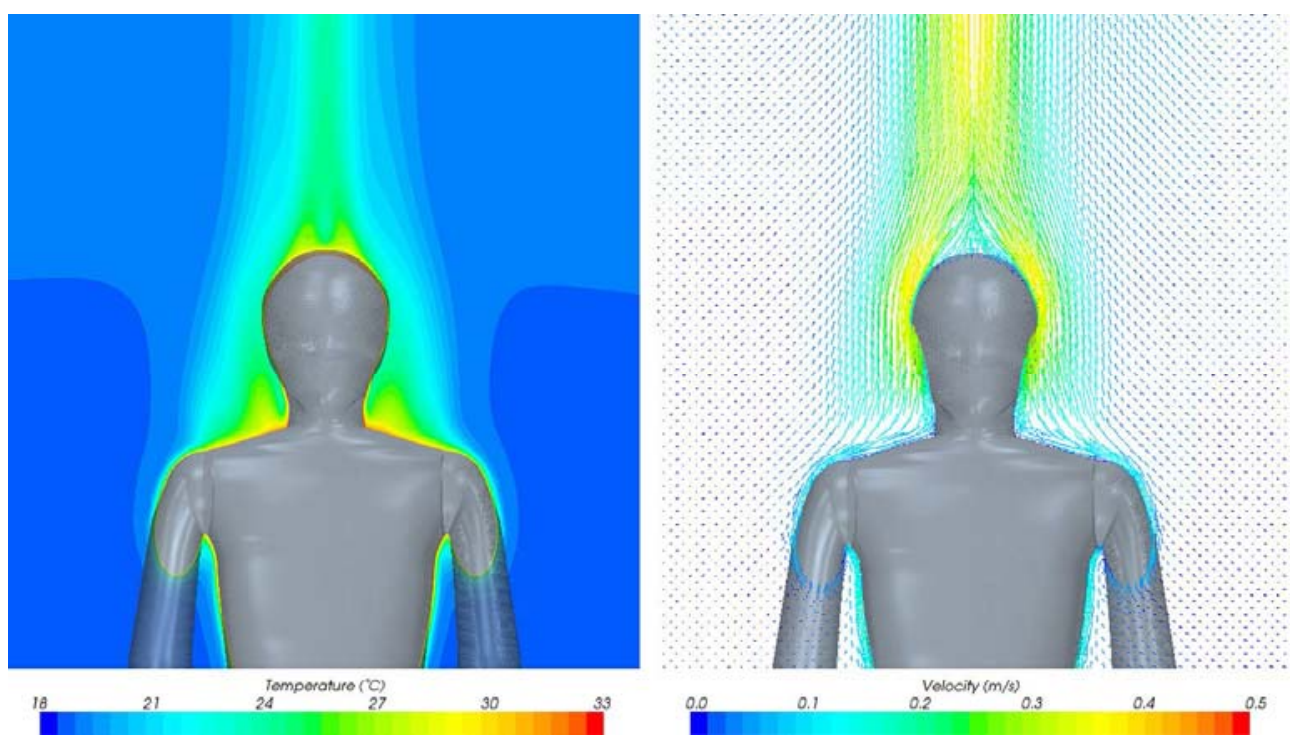

Figure 4 Simulated air temperature and velocity in the microclimate when air temperature is set to $\theta_{a}=18^{\circ} \mathrm{C}$ 
$=35 \mathrm{~W} / \mathrm{m}^{2}$ was assigned to the body surface. The results show that using this approach can lead to unrealistically high surface temperature in some areas. Therefore, the simulated air temperature should be transferred to the thermoregulation model instead of heat flux.

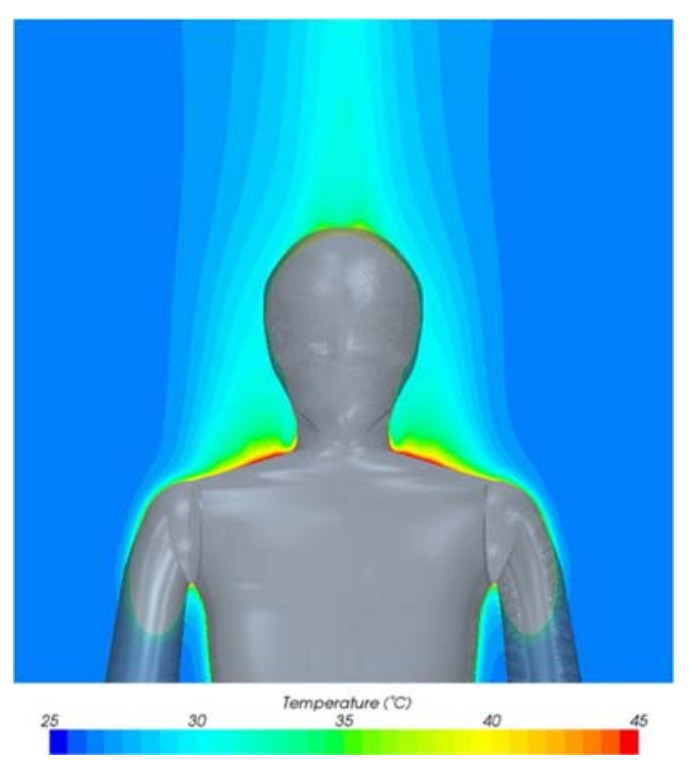

Figure 5 Unrealistic air temperature when defining the boundary conditions using heat flux

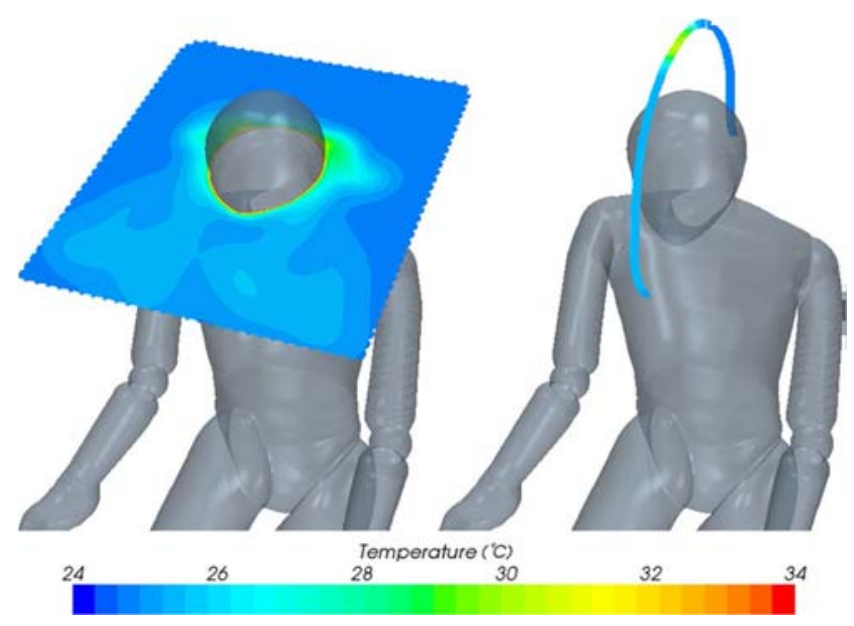

Figure 6 Air temperature around the head in a plane (left), and an isodistance (right)

Multiple methods were tested to determine the thickness of the human body's microclimate and the location of the macroclimate around each body segment. The first method was to create a two-dimensional plane or a threedimensional volume to capture the temperature distribution around the body segment in question. Figure 6 (left) shows an example of this technique. However, automated determination of air temperature in the macroclimate is difficult to achieve using this approach as the microclimate is too non-uniformly shaped. Another method, shown on the right side in Figure 6, determines air temperature over the mean of an isodistance line or area. This method has the drawback of including the values of disturbed regions (the plume in this example) in the results too.

Therefore, a different approach was used in this study to determine the simulated air temperature in the macroclimate around the respective body segment. In the CFD simulation, the air temperature was taken orthogonally to the surface of each segment (Figure 7). The air temperature in the macroclimate is determined using an algorithm from the temperature profile curve. Figure 8 shows the approach of determining the stopping criterion (e.g. $\varepsilon=0.1 \mathrm{~K}$ ) on the basis of the difference between predecessor and successor using:

$$
\varepsilon \geq \theta_{a}\left(x_{i-1}\right)-\theta_{a}\left(x_{i}\right)
$$

However, it is important to incorporate multiple predecessors to minimize errors caused by any temperature irregularities in the CFD simulation.

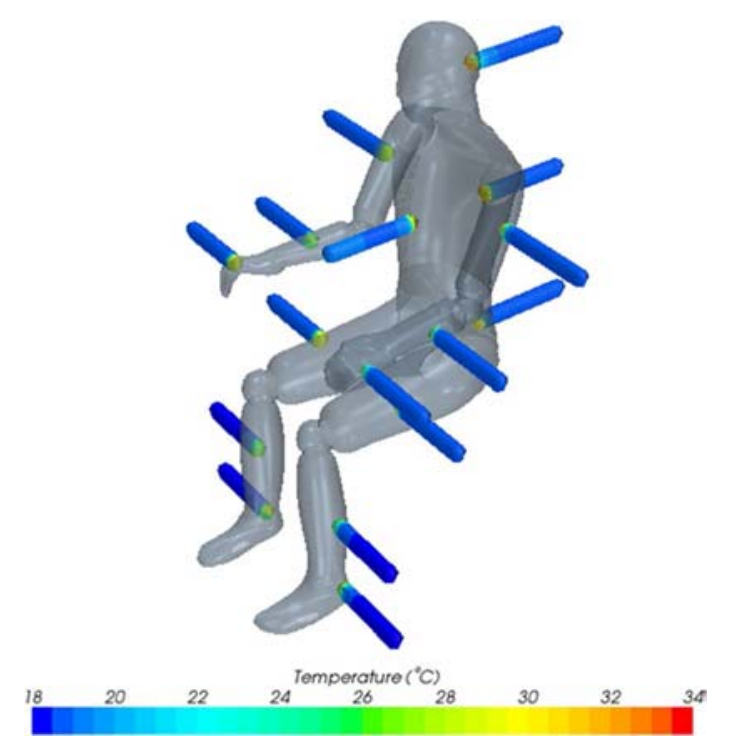

Figure 7 Air temperature taken orthogonally to the surface

Furthermore, a function approximation was derived using simulation results of the microclimate under different boundary conditions to reduce uncertainties. The following exponential function showed a good agreement $\left(R^{2}=0.995 ; \sigma\right.$ $=0.01$ ) in the curtail area of the transition from the microclimate to the macroclimate (Figure 9):

$$
\theta_{a}(x)=A+B \cdot e^{C x}
$$

The coefficients $A, B$, and $C$ were determined using the method of least squares for each segment under each boundary condition. The derivation of this function can be used to determine the point of the curve at which the slope corresponds to the termination criterion (for example, $\varepsilon=0.1$ $\mathrm{K} / \mathrm{m})$ :

$$
\theta_{a}^{\prime}(x) \leq \varepsilon
$$




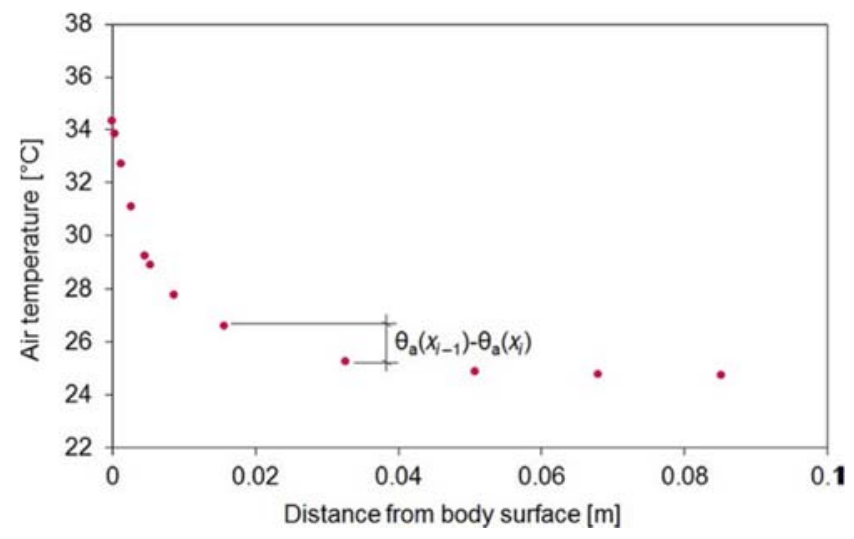

Figure 8 Simulated air temperature in the vicinity of the body at $24^{\circ} \mathrm{C}$

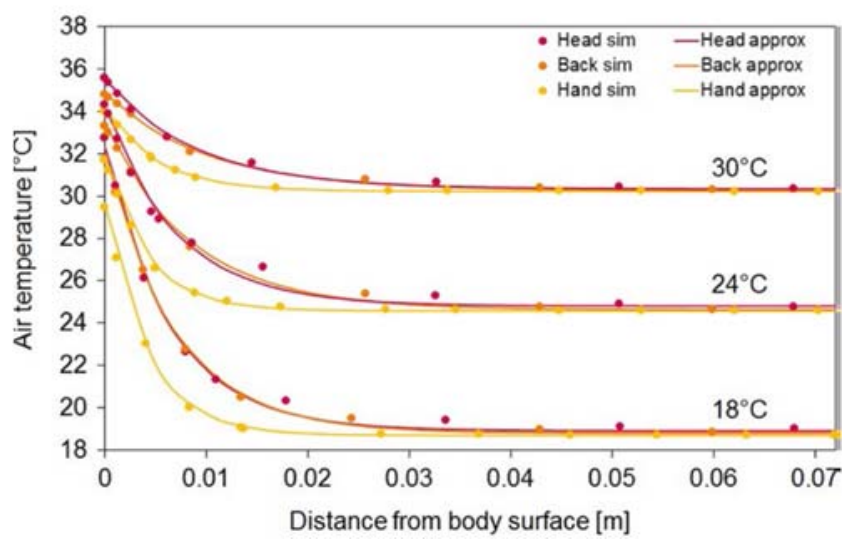

Figure 9 A good agreement of the approximation with the simulation results for three body segments as an example under three air temperature setups $\left(\theta_{a}=18,24\right.$, and $\left.30^{\circ} \mathrm{C}\right)$

In addition to air temperature, the air velocity is often transferred to the thermoregulation model during the coupling with CFD simulation to determine the convective heat transfer coefficient $h_{c}{ }^{34}$ In the UCB model, the convective heat transfer coefficient $h_{c}$ is defined as a function of the air velocity using equation (4).

$$
h_{c}=b \cdot v_{a}^{n}
$$

Where $b$ and $n$ are experimentally determined, segment-dependent coefficients. In de Dear's study,34 a uniform temperature gradient between surface and ambient $(\Delta \theta=12 \mathrm{~K})$ was assumed. However, our measurements and simulations showed that the experimental conditions cannot always be generalized and transferred to other boundary conditions. Table 1 shows that there is a dependence between the room climate and the heat transfer coefficients. The results would be even more different if the heat transfer of the human body included forced convection in addition to free convection. This confirms the assumption that both simulated and measured heat transfer coefficients should not be generalized. Therefore, the heat transfer coefficients were calculated directly from the CFD simulation using:

$$
h_{c}=\frac{q}{\theta_{s}-\theta_{a}}
$$

Whereas the heat flux $q$ is known from the CFD simulation, air temperature $\theta_{a}$ is determined using equations $1-3$, and skin temperature $\theta_{s}$ is determined by the UCB model. When the surrounding environment is strongly inhomogeneous, the heat transfer coefficients can be determined using the temperature in the near-wall cell.

This approach determines the heat transfer coefficients according to the given climatic conditions instead of the velocity of the flow. Therefore, it increases the accuracy of the calculations and avoids errors in the non-trivial determination of the air velocity around the segment.

TABLE 1 Simulated heat transfer coefficients

\begin{tabular}{llll}
\hline $\begin{array}{l}\text { Body } \\
\text { segment }\end{array}$ & \multicolumn{3}{l}{ Heat transfer coefficient $\boldsymbol{h}_{\boldsymbol{c}}\left[\mathbf{W} / \mathbf{m}^{\mathbf{2}} \mathbf{K}\right]$} \\
\cline { 2 - 4 } & $\mathbf{1 8}^{\circ} \mathbf{C}$ & $\mathbf{2 4}^{\circ} \mathbf{C}$ & $\mathbf{3 0}^{\circ} \mathbf{C}$ \\
\hline Head & 3.52 & 3.64 & 3.26 \\
Chest & 2.39 & 2.12 & 1.79 \\
Back & 2.91 & 2.56 & 2.29 \\
Pelvis & 3.23 & 2.71 & 2.35 \\
Upper arm & 3.25 & 2.97 & 2.53 \\
Lower arm & 4.04 & 3.57 & 3.11 \\
Hand & 4.53 & 3.90 & 3.49 \\
Thigh & 3.32 & 2.67 & 2.27 \\
Leg & 2.69 & 2.44 & 2.15 \\
Foot & 10.56 & 7.13 & 6.69 \\
Average & $\mathbf{3 . 7 4}$ & $\mathbf{3 . 1 2}$ & $\mathbf{2 . 7 4}$ \\
\hline
\end{tabular}

\section{Example of the coupling applications}

Radiant floor cooling was investigated as a practical application of the coupling of CFD simulation and the UCB model to study thermal comfort. Since space cooling can be a significant part of the energy consumption of buildings, the use of energy-efficient systems is becoming increasingly important. Compared to conventional methods, surface cooling is able to drastically reduce energy consumption. Depending on various parameters, a savings potential of 10$80 \%$ compared to conventional air-conditioning systems was reported in literature. ${ }^{35,36}$ Furthermore, radiant cooling can result in improved thermal comfort compared to all-air systems. ${ }^{37}$

The same geometry and solver setup used in the coupling investigations was implemented in the floor cooling simulation. Three air exchange rates were investigated $(n=0$, $\left.2.3,4.7 \mathrm{~h}^{-1}\right)$. The no-ventilation case $\left(n=0 \mathrm{~h}^{-1}\right)$ was examined to simulate a room with no mechanical ventilation system and a radiant floor heating setup, which is common in Germany and other regions with mild summers and cold winters. Thus, the already-existing floor heating setup can be implemented for 
floor cooling by circulating cold water through the tubing embedded in the floor instead of hot water.

Table 2 shows the boundary conditions of the simulated cases. A total of 12 different scenarios were selected, the temperature of the walls was assigned equal to the inlet air temperature. Hence, cooling is guaranteed by the floor cooling which was set to a surface temperature of $19^{\circ} \mathrm{C}$ during all simulation cases. A lower temperature allows for a higher cooling load, but it results in a higher dissatisfaction vote $\left(\theta_{\text {floor }}\right.$ $=19^{\circ} \mathrm{C}$ corresponds to $\left.P D=10 \%\right) .38$

TABLE 2 Simulated cases during the floor cooling study

\begin{tabular}{lllll}
\hline Case No. & $\boldsymbol{\theta}_{\text {floor }}\left[{ }^{\circ} \mathbf{C}\right]$ & $\boldsymbol{\theta}_{\boldsymbol{w}}\left[{ }^{\circ} \mathbf{C}\right]$ & $\boldsymbol{v}_{\text {inlet }}[\mathbf{m} / \mathbf{s}]$ & $\boldsymbol{n}\left[\mathbf{h}^{-1}\right]$ \\
\hline 1 & 24 & 0 & 0 \\
2 & 26 & 0 & 0 \\
3 & 28 & 0 & 0 \\
4 & 30 & 0 & 0 \\
5 & 24 & 1.2 & 2.3 \\
6 & 26 & 1.2 & 2.3 \\
7 & 19 & 28 & 1.2 & 2.3 \\
8 & & 1.2 & 2.3 \\
9 & 24 & 2.5 & 4.7 \\
10 & 26 & 2.5 & 4.7 \\
11 & 28 & 2.5 & 4.7 \\
12 & 30 & 2.5 & 4.7 \\
\hline
\end{tabular}

Thermal comfort was determined for the whole body (overall thermal comfort) and for each body segment (local thermal comfort) using the UCB model. The metabolic activity of the user was defined as 1 met (standard Stolwijk). ${ }^{39}$ The relative humidity, which is not taken into account by the CFD simulation, was set to the default value of $\varphi=50 \%$. An average office attire was chosen in the simulation. This attire consisted of a shirt, light jacket, pants, shoes, socks, under shirt, and under pants. Since an accurate clothing insulation value is essential to evaluate thermal comfort, it was necessary to consider the coverage degree of each clothing item when estimating the clo value input in the UCB model. Therefore, the whole body clo values for each attire item reported by McCullough et al. ${ }^{40}$ were converted to the segmentation of the UCB model. This was performed using covering percentages provided by McCullough et al.40 Superimposed garments were summed using the following equation:

$$
I_{c l}=\sum_{i=1}^{n} I_{c l u, i}
$$

Where $I_{c l}$ is the total clothing insulation value at the segment, $I_{c l u}$ is the insulation value of the clothing layer. In addition, a direct contact with the floor has been assigned to the feet or shoes. Thus, the effects of heat conduction, which are vital particularly in the case of floor cooling, can be traced. The insulation value of the chair was neglected in this simulation.

Figure 10 (left) shows the temperature profile for simulation case No. 2 in which the ventilation system was off $\left(n=0 \mathrm{~h}^{-1}\right)$. The convective heat emission from the human body was the only cause for the mass transport. Hence, there is no uniform distribution of the cooling load due to the lack of air mixing. This resulted a distinct vertical temperature gradient with a temperature difference of $\Delta \theta_{a}=7 \mathrm{~K}$ between the floor surface and the air temperature at head height. The investigation of the overall thermal comfort and sensation using the UCB model exhibited further weaknesses. Overall thermal comfort score was barely comfortable $\left(C_{o}=0.34\right)$, and the overall sensation score was slightly warm $\left(S_{o}=0.8\right)$. This is due to the large difference in air temperature between the head and the feet.

The right-side figure in Figure 10 shows the simulation results of the same boundary conditions with the ventilation system activated (case No. $6, n=2.3 \mathrm{~h}^{-1}$ ). Air was supplied from an inlet opening located close to the floor level to create a displacement ventilation effect. Thus, it ensured a better distribution of the cooling load in the room despite the supply of warm air. Moreover, the heat transfer coefficient of the floor is increased when ventilation is used. Hence, the heat gain can

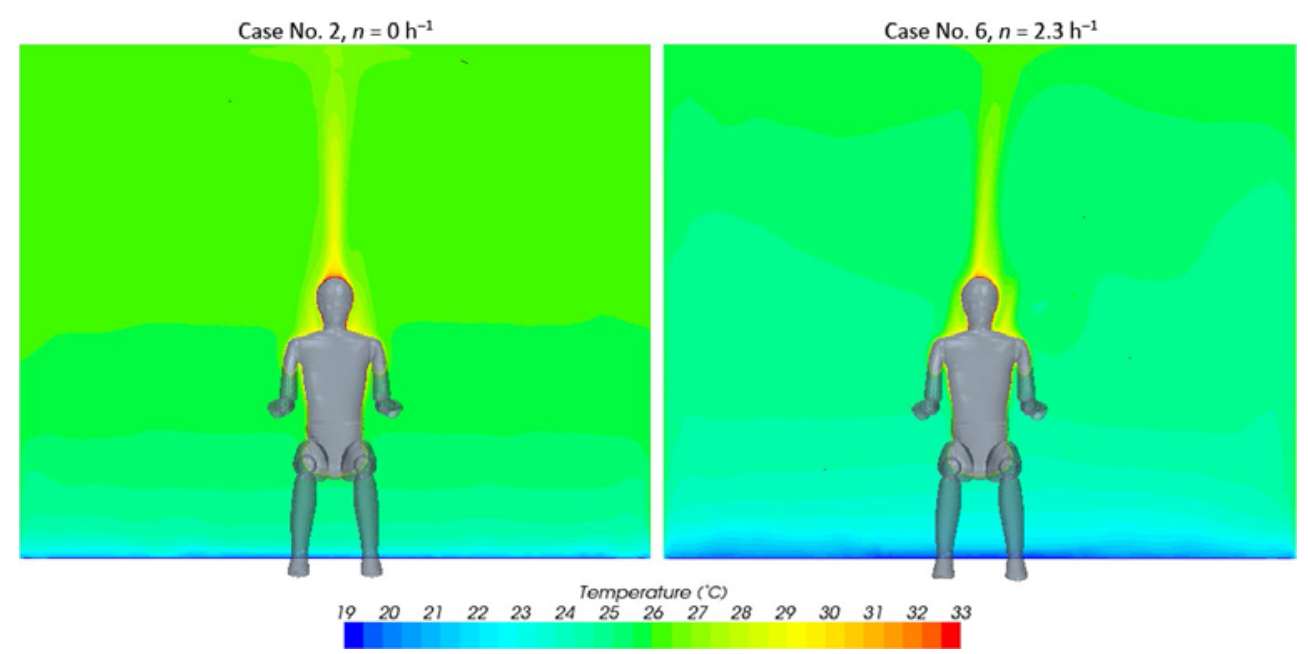

Figure 10 Simulated profiles of air temperature when floor cooling is implemented 
be compensated by the supplied air. The vertical temperature gradient dropped to $\Delta \theta_{a}=6 \mathrm{~K}$ when implementing the ventilation system. Overall comfort improved slightly $\left(C_{o}=\right.$ $0.83)$, and overall sensation approached the neutral vote $\left(S_{o}=\right.$ 0.50 ). When the air exchange rate was increased to $4.7 \mathrm{~h}^{-1}$, vertical temperature gradient was slightly increased. Overall comfort and sensation were at an intermediate level between the other two simulated cases as well. This is due to high heat gain caused by the supplied air, which in this case can no longer be compensated by the floor cooling. However, when the air temperature difference between the ankle $(0.1 \mathrm{~m})$ and the head $(1.1 \mathrm{~m})$ is determined according to DIN EN ISO 7730,38 the result is completely different. The case with no ventilation should have a gradient of $\Delta \theta_{a}=1.7 \mathrm{~K}$, which increases to $\Delta \theta_{a}=2.4 \mathrm{~K}$ when ventilation is implemented at $n$ $=2.3 \mathrm{~h}^{-1}$ and $\Delta \theta_{a}=2.7 \mathrm{~K}$ at $n=4.7 \mathrm{~h}^{-1}$. According to this standard, the room without ventilation with category A $\left(\Delta \theta_{a}<\right.$ $2 \mathrm{~K}$ ) is higher than with ventilation (category $\mathrm{B}, \Delta \theta_{a}<3 \mathrm{~K}$ ). This is due to the high temperature gradient at the first ten centimetres above the floor, which is not taken into account by the standardized approach. The suitability of this standardized approach can therefore be called into question, at least for the determination of thermal comfort in the case of floor cooling.

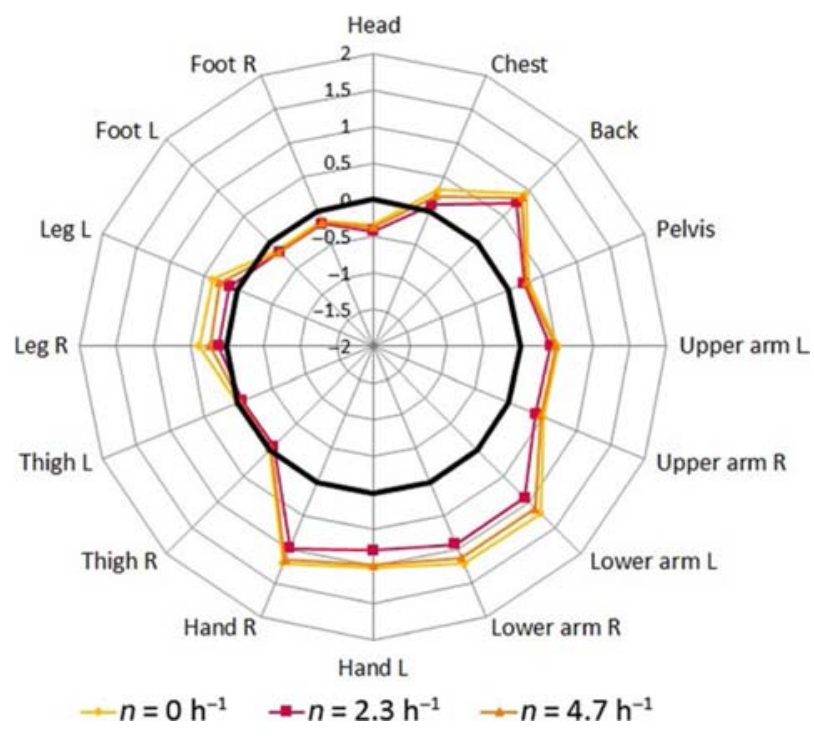

Figure 11 Local thermal sensation during the three simulated air exchange rates (cases No. 2, 6, and 10)

Local thermal sensation and comfort were determined based on local climatic conditions around each body segment. Figure 11 shows the thermal sensation of the individual body segments under the three tested air exchange rates and walls setting temperature of $\theta_{w}=26^{\circ} \mathrm{C}$ (cases No. 2, 6, and 10), taking into account each segment with its own coefficients. While the lower half of the body has a nearly neutral feeling, the upper half of the body was perceived as warm due to the vertical temperature gradient. This was slightly improved when the ventilation system is used. Figure 12 shows the local thermal comfort of body segments, which is dependent on their respective local sensation. ${ }^{27}$ For example, the hand was perceived as warm, which leads to the evaluation of "just comfortable." On the contrary, the sensation at the head was "slightly cool," which was nevertheless evaluated as comfortable. This is due to the thermosensitivity of the head which has high sensitivity to heat, but less sensitivity to cold.

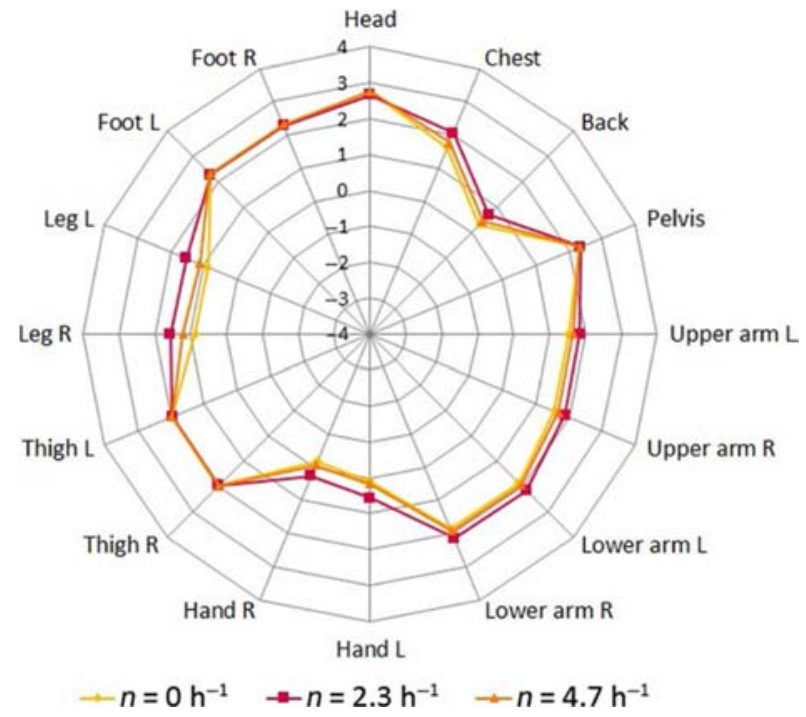

Figure 12 Local thermal comfort during the three simulated air exchange rates (cases No. 2, 6, and 10)

\section{Conclusions, limitations, and future research}

This research presents an approach for automated coupling of CFD simulation and the UCB thermal comfort model. The CFD simulation was used to determine the climatic conditions of the indoor environment, which were transferred to the UCB model. The UCB model then calculates the surface temperature of each body segment and sends it back to the CFD solver in an iterative loop. Since the accuracy of the transferred parameters of the environment surrounding the human body is vital for the accuracy of the coupling, a new approach was developed to determine the transferred values instead of the simplified approaches presented in literature. This approach implemented an algorithm to calculate the value of air temperature in the macroclimate based on the temperature curve using the difference between predecessor and successor. The developed model represents a robust tool that functions independently from the boundary conditions. It can be used in various applications to calculate thermal comfort.

The conclusions reported above should be considered in the light of several limitations that this study encountered. These limitations suggest topics to be addressed in future research. A significant limitation was the lack of computational power large enough to conduct transient state simulation within a reasonable span of time, since both the CFD solver and the UCB model offer the option of running transient simulation. Another limitation was implementing a naked 
manikin in the CFD model to simplify the simulation. Even though clothing was accounted for in the UCB model, the manikin geometry in the CFD solver needs to be clothed too because clothing significantly changes the characteristics of the body's microclimate.

As the presented model accounts for heat dissipation from the human body through convection and radiation only, future research is needed to include heat loss via evaporation and respiration in the CFD model as these mechanisms play an important role in human thermoregulation. Furthermore, including the breathing process in the CFD simulation allows studying its influence on the body's microclimate, and consequently its influence on the coupling model.

\section{Acknowledgements}

The authors would like to show their gratitude to the German Federal Ministry for Education and Research (BMBF) and the German Academic Exchange Service (DAAD) for their constant support. Additionally we highly appreciate the cooperation with the Center for the Built Environment, UC Berkeley.

\section{References}

1. Melikov AK. Human body micro-environment: The benefits of controlling airflow interaction. Build Environ. 2015;91:70-77.

2. Licina D, Melikov A, Pantelic J, Sekhar C, Tham KW. Human convection flow in spaces with and without ventilation: personal exposure to floor-released particles and cough-released droplets. Indoor Air. 2015;25(6):672-682.

3. Licina D, Melikov A, Sekhar C, Tham KW. Human convective boundary layer and its interaction with room ventilation flow. Indoor Air. 2015;25(1):21-35.

4. Licina D, Melikov AK, Sekhar C, Tham KW. Interaction of convective flow generated by human body with room ventilation flow: impact on transport of pollution to the breathing zone. Proceedings of Indoor Air 2014, Hong Kong, 2014: 413-420.

5. Voelker C, Maempel S, Kornadt O. Measuring the human body's microclimate using a thermal manikin. Indoor Air. 2014;24(6):567-579.

6. Licina D, Melikov A, Sekhar C, Tham KW. Air temperature investigation in microenvironment around a human body. Build Environ. 2015;92:39-47.

7. Licina D, Pantelic J, Tham KW, Sekhar C. Experimental characterization of the human convective boundary layer in a calm indoor environment. Proceedings of ASHRAE IAQ 2013: Environmental Health in Low Energy Buildings, Vancouver, Canada, 2013.

8. Licina D, Pantelic J, Melikov A, Sekhar C, Tham KW. Experimental investigation of the human convective boundary layer in a quiescent indoor environment. Build Environ. 2014;75:79-91.

9. Zukowska D, Melikov A, Popiolek Z. Impact of personal factors and furniture arrangement on the thermal plume above a sitting occupant. Build Environ. 2012;49:104116.

10. Murakami S, Kato S, Zeng J. Flow and temperature fields around human body with various room air distribution, CFD study on computational thermal manikin part 1 . ASHRAE Trans. 1997;103(1):3-15.

11. Murakami S. CFD study on the microclimate around the human body with inhalation and exhalation. Proceedings of Roomvent 2002, Copenhagen, Denmark, 2002: 23-35.

12. Maué J, Wahl D, Currle J. Computation of the Thermal Environment in Passenger Compartments and Evaluation of Thermal Comfort. Proceedings of Comfort in the automotive industry-Recent developments and achievements, Bologna, Italy, 1997: 147-155.

13. Murakami S, Kato S, Zeng J. Combined simulation of airflow, radiation and moisture transport for heat release from a human body. Build Environ. 2000;35(6):489-500.

14. Tanabe S, Kobayashi K, Nakano J, Ozeki Y, Konishi M. Evaluation of thermal comfort using combined multinode thermoregulation (65MN) and radiation models and computational fluid dynamics (CFD). Energ Buildings. 2002;34(6):637-646.

15. Bartsch, P, Spille-Kohoff A. Simulation der MenschRaumklima-Wechselwirkung mit dem Tanabe-Modell [Simulation of the interaction between Room Air Flow and Human Body Using the Tanabe Model]. KI Luft- und Kältetechnik. 2006;42(9):359-364.

16. Streblow R, Mueller D, Gores I, Bendfeldt P. Prediction of thermal sensation using an optimized 33 node simulation model. Proceedings of Roomvent 2009, Busan, South Korea, 2009:1047-1053.

17. Cropper PC, Yang T, Cook MJ, Fiala D, Yousaf R. Exchange of simulation data between CFD programmes and a multisegmented human thermal comfort model. Proceedings of the 5th Windsor Conference, Air conditioning and the low carbon cooling challenge, Windsor, UK, 2008.

18. van Treeck C, Wenisch P, Borrmann A, Pfaffinger M, Wenisch O, Rank E. ComfSim - Interaktive Simulation des thermischen Komforts in Innenräumen auf Höchstleistungsrechnern [Interactive simulation of thermal comfort in indoor spaces using highperformance computers]. Bauphysik. 2007;29(1):2-7.

19. Huang L. First vehicle passenger thermal comfort model helps optimize HVAC systems. FLUENT Inc. 2002.

20. Gao N, Niu J, Zhang H. Coupling CFD and Human Body Thermoregulation Model for the Assessment of Personalized Ventilation. HVAC\&R Res. 2006;12(3):497518.

21. Gao NP, Zhang H, Niu JL. Investigating Indoor Air Quality and Thermal Comfort Using a Numerical Thermal Manikin. Indoor Built Environ. 2007;16(1):7-17. 
22. Huizenga C, Hui Z, Arens E. A model of human physiology and comfort for assessing complex thermal environments. Build Environ. 2001;36(6):691-699.

23. Voelker C, Hoffmann S, Kornadt O, Arens E, Zhang H, Huizenga C. Heat and moisture transfer through clothing. Proceedings of the 11th IBPSA Conference, Glasgow, Scotland, 2009:1360-1366.

24. Zhang H, Huizenga C, Arens E, Yu T. Considering individual physiological differences in a human thermal model. J Therm Biol. 2001;26(4-5):401-408.

25. Zhao Y, Zhang H, Arens EA, Zhao Q. Thermal sensation and comfort models for non-uniform and transient environments, part IV: Adaptive neutral setpoints and smoothed whole-body sensation model. Build Environ. 2014;72:300-308.

26. Koelblen B, Psikuta A, Bogdan A, Annaheim S, Rossi RM. Thermal sensation models: a systematic comparison. Indoor Air. 2017;27(3):680-689.

27. Alsaad H, Voelker C. CFD Assessment of Thermal Comfort and Indoor Air Quality Using Ductless Personalized Ventilation. Proceedings of the 15th IBPSA Conference, San Francisco, CA, USA, 2017:113-121.

28. Alsaad H, Voelker C. Performance Comparison of a Ductless Personalized Ventilation System and an Axial Fan Using CFD Simulations. Proceedings of HB2017Europe, Lublin, Poland, 2017.

29. Zhang H, Arens E, Huizenga C, Han T. Thermal sensation and comfort models for non-uniform and transient environments, part I: Local sensation of individual body parts. Build Environ. 2010;45(2):380-388.

30. Zhang H, Arens E, Huizenga C, Han T. Thermal sensation and comfort models for non-uniform and transient environments, part II: Local comfort of individual body parts. Build Environ. 2010;45(2):389-398.

31. Zhang H, Arens E, Huizenga C, Han T. Thermal sensation and comfort models for non-uniform and transient environments, part III: Whole-body sensation and comfort. Build Environ. 2010;45(2):399-410.
32. Bohl W, Elmendorf W. Technische Strömungslehre [Technical Fluid Mechanics]. 13. Aufl. Würzburg: Vogel Verlag; 2005. Online-Resource. ISBN: 9783834330291.

33. Cropper P, Yang T, Cook M, Fiala D, Yousaf R. Coupling a human thermal comfort model with computational fluid dynamics to predict thermal comfort in naturally ventilated environments. $J$ Build Perform Simu. 2009;3(3):233-243.

34. de Dear RJ, Arens E, Hui Z, Oguro M. Convective and radiative heat transfer coefficients for individual human body segments. Int J Biometeorol. 1997;40(3):141-156.

35. Imanari $\mathrm{T}$, Omori $\mathrm{T}$, Bogaki $\mathrm{K}$. Thermal comfort and energy consumption of the radiant ceiling panel system: Comparison with the conventional all-air system. Energ Buildings. 1999;30(2):167-175.

36. Memon RA, Chirarattananon S, Vangtook P. Thermal comfort assessment and application of radiant cooling: A case study. Build Environ. 2008;43(7):1185-1196.

37. Karmann C, Schiavon S, Bauman F. Thermal comfort in buildings using radiant vs. all-air systems: A critical literature review. Build Environ. 2017;111:123-131.

38. DIN EN ISO 7730. Ergonomie der thermischen Umgebung - Analytische Bestimmung und Interpretation der thermischen Behaglichkeit durch Berechnung des PMVund des PPD- 140 Indexes und Kriterien der lokalen thermischen Behaglichkeit [Ergonomics of the thermal environment - Analytical determination and interpretation of thermal comfort by calculation of the PMV and PPD 140 index and criteria of local thermal comfort]: Deutsches Institut für Normung; 2006.

39. Stolwijk J. A mathematical model of physiological temperature regulation in man. Washington, D.C.: NASA Contractor Reports CR-1855; 1971.

40. McCullough EA, Jones BW, Huck J. A comprehensive data base for estimating clothing insulation. ASHRAE Trans. 1985;91(2A):29-47. 